\title{
DESARROLLO DE EXPERIMENTOS VIRTUALES BASADOS EN ADOBE FLASH
}

\author{
DEVELOPMENT OF VIRTUAL EXPERIMENTS BASED ON \\ ADOBE FLASH
}

\author{
Comina Bellido German Yuri ${ }^{1}$
}

\begin{abstract}
RESUMEN
El presente trabajo describe el desarrollo de dos experimentos virtuales usando Adobe Flash. El primero consta de un sistema masa-resorte el cual permite a los estudiantes hallar el valor de la gravedad terrestre con la incertidumbre correspondiente. El segundo consta de un sistema compuesto por tres masas y cuatro resortes. Éste experimento permite al alumno visualizar movimientos de sistemas mecánicos complejos, además de darle una visión acerca de la mecánica lagrangeana, así como de los conceptos de valor propio y estado propio de un sistema.
\end{abstract}

Ambos programas son interactivos, permitiendo a los alumnos observen cambios en el sistema físico a medida que realicen cambios en los parámetros correspondientes. Estos experimentos virtuales pueden ser utilizados como complementos en las clases teóricas incrementando el dinamismo en las mismas. Los programas pueden correr directamente desde las computadoras o pueden instalarse en un servidor y ser ejecutados desde cualquier parte del mundo por clases enteras al mismo tiempo, sin importar el sistema operativo que usen.

Palabras claves. - Incertidumbre, Sistema mecánico, Mecánica lagrangiana.

\begin{abstract}
In this work the development of two virtual experiments using Adobe Flash has been reported. The first virtual experiment is based on a mass-spring mechanical system, with which the students can calculate the value of earth gravity, including the measurement error. The second virtual experiment helps students to visualize complex mechanical movements, and also lead them in to the concept of lagrangian mechanics as well as eigen values, vectors and eigen states of a system. In this experiment the mechanical system is composed by three masses and four springs.

Both programs are interactive, so the students can change different parameters and therefore visualize changes in the physical ensemble. This fact enables physic teachers to use them as learning tools in an interactive class. The compiled programs can run standalone using free Adobe Flash player or through a webpage. In this last case, students from all over the world can use these tools at the same time.
\end{abstract}

Key words. - Uncertainty, Mechanical system, Lagrangian mechanics.

\footnotetext{
La Revista Científica TECNIA protege los derechos de autor bajo la Licencia 4.0 de Creative Commons: Attribution 4.0 International (CC BY 4.0)

${ }^{1}$ Docente de la Escuela de Ing Física, Facultad de Ciencias UNI
} 


\section{INTRODUCCIÓN}

Desde hace varios años, investigadores en la enseñanza de Física han sostenido que las clases tradicionales de los cursos de Física Básica, en donde solo hay estudiantes pasivos, poseen limitaciones en el aprendizaje del concepto que se simulaciones en computadora [6]. De hecho la aparición de la computadora no solo revolucionó la investigación en física, creando la disciplina de la Física Computacional, sino que además permitió la implementación efectiva de la educación interactiva a través de varios programas de simulación [7-10].

Adobe Flash® es una herramienta de edición que permite crear documentos, que posean gran capacidad de interacción con el usuario. Los proyectos de Flash pueden abarcar desde simples animaciones hasta contenido de vídeo, presentaciones complejas, aplicaciones y cualquier otra utilidad relacionada. Los fragmentos independientes de contenido creados con Flash se denominan aplicaciones, aunque se trate solamente de una animación básica.

ActionScript es el código que permite añadir interactividad a los elementos multimedia del documento Flash. Por ejemplo se puede utilizar ActionScript para modificar el tamaño, posición, ángulo de rotación, color, etc. De figuras o letras en el documento Flash.

En el presente trabajo se ha aprovechado ésta particularidad de programa Adobe Flash ${ }^{\circledR}$ para construir dos experimentos virtuales.

El primero tiene como objetivo determinar el valor de la gravedad terrestre usando una masa que cuelga de un resorte, el cual tiene un extremo unido al techo. La programación de éste experimento virtual es simple. Este experimento se utilizó en un laboratorio del curso de Tratamiento Estadístico de Datos (IF-232), en la Facultad de Ciencias de la Universidad Nacional de Ingeniería. Allí los alumnos tomaron varias mediciones para hallar la gravedad con el valor de incertidumbre correspondiente.

El segundo es una simulación de tres masas, unidas por cuatro resortes, donde los extremos del primer y último resorte están unidos a dos paredes inamovibles. El sistema de tres masas y cuatro trata [1,2]. El uso de elementos interactivos incrementa la efectividad para entender los conceptos tratados, muy por encima del método tradicional [3-5].

La educación interactiva de la física puede implementarse en forma satisfactoria usando resortes se mueve horizontalmente sobre un solo eje de movimiento. Éste modelo se utilizó para la presentación del tema "Resortes: Oscilaciones Propias" en el curso de Mecánica Clásica en la Escuela de Post-Grado de la Facultad de Ciencias en la Universidad Nacional de Ingeniería.

\section{FUNDAMENTO TEÓRICO}

Ambos sistemas se considerarán pequeños respecto al tamaño de la tierra, por tanto el valor de la gravedad es constante en la región geométrica donde se desarrolla el experimento. Además, el techo para el primer experimento y las paredes en el segundo están unidos perfectamente a la tierra, se mueven con ella. El tiempo que transcurre durante el desarrollo del experimento es pequeño respecto al tiempo de rotación de la tierra; respecto a su eje ( 24 horas) y respecto al sol (365 días), por ello se considerará que la tierra no rota, por tanto se puede tomar al techo para el primer experimento y a las paredes en el segundo como referencias inerciales.

Para el primer sistema se tiene un cuerpo de masa "M", por comodidad se elegirá una esfera maciza, que cuelga de un resorte de constante " $\mathrm{K}$ " $\mathrm{y}$ longitud natural " $\mathrm{X}_{0}$ ". Como se muestra en la Figura 1(a).
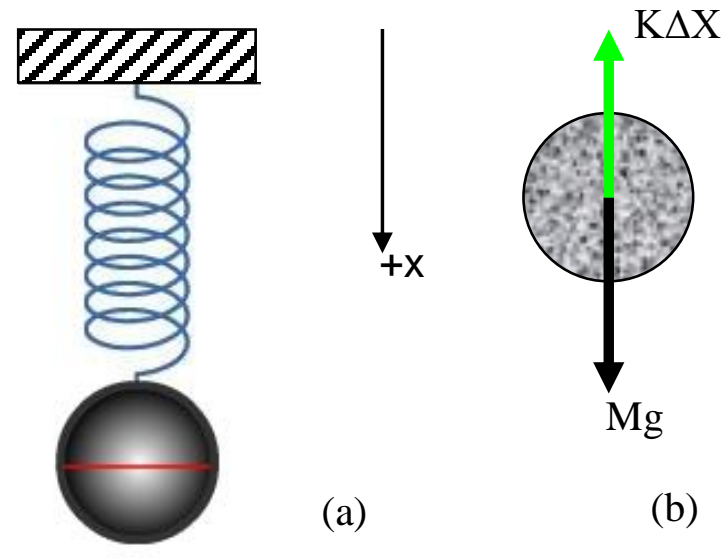

(a)

(b)

Figura 1. (a) Primer sistema masa que cuelga de un resorte. (b) Diagrama de cuerpo libre para la esfera. 
Considerando que el valor de la gravedad es "g" y

la elongación del resorte es $\Delta \mathrm{X}=\mathrm{X}-\mathrm{X}_{0}$, del

diagrama de cuerpo libre para la esfera se tendrá:

$$
X=X_{0}+\frac{M g}{K}
$$

El segundo sistema consta de tres masas y cuatro resortes como se muestra en la Figura 2.

L

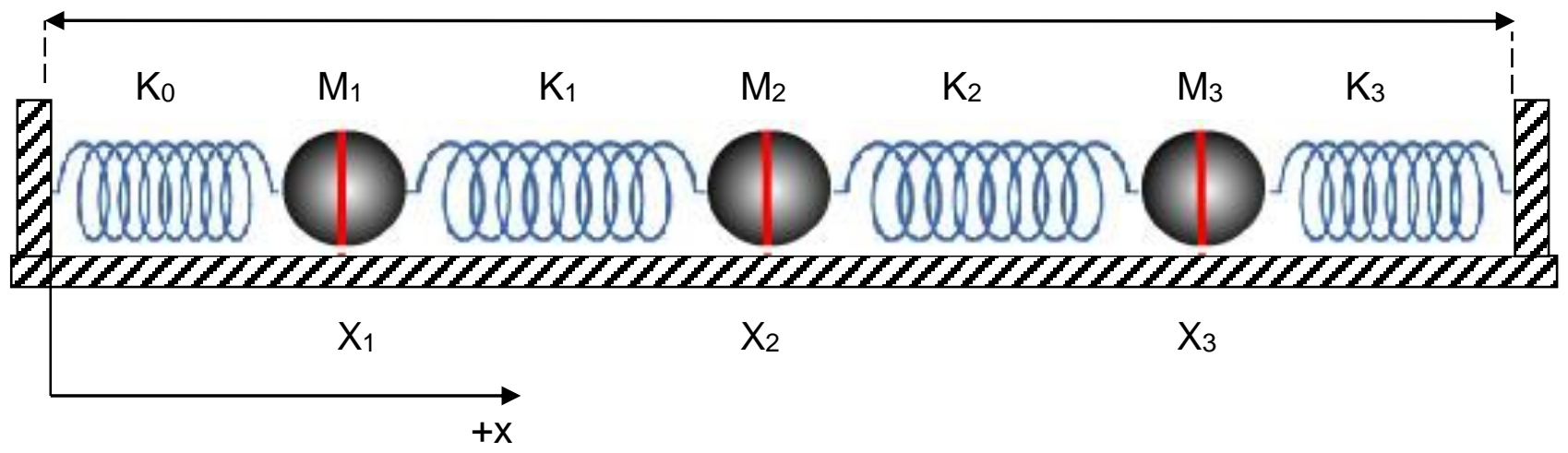

Figura 2. Sistema de tres masas y cuatro resortes.

Las masas, que por comodidad se considerarán esferas, pueden moverse sin fricción y los resortes nunca se doblan. Estas consideraciones se pueden implementar experimentalmente sujetando a cada esfera por una cuerda larga al techo, todas deberán tener la misma altura y los resortes deberán permanecer horizontales. Además, experimentalmente los estiramientos iniciales deberán ser comparables con las longitudes naturales de los resortes y sobre el mismo eje horizontal del movimiento del sistema.

La longitud total del sistema es "L". Se considerará que todas las esferas tienen la misma masa "M" y todos los resortes tienen la misma constante elástica "K".

El Lagrangiano del sistema es:

$\mathrm{L}\left(\mathrm{X}_{1}, \mathrm{X} 2, \mathrm{X} 3, \mathrm{q} 1, \mathrm{q} 2, \mathrm{q} 3\right)=$

$\frac{M_{1} q_{1}^{2}}{2}+\frac{M_{2} q_{2}^{2}}{2}+\frac{M_{3} q_{3}^{2}}{2}-\frac{1}{2} \sum_{j=0}^{3} K_{j}\left(\left(X_{j+1}-X_{0 j+1}\right)-\left(X_{j}-X_{0 j}\right)\right)^{2}$

Donde $\mathrm{q}_{\mathrm{j}}$ son variables que luego sobre la trayectoria se convertirán en las velocidades de las esferas. $\mathrm{X}_{0 \mathrm{j}+1}$ y $\mathrm{X}_{0 \mathrm{j}}$ son las posiciones iniciales de las masas $\mathrm{j}+1 \mathrm{y}$ j respectivamente. $\mathrm{X}_{0}$ sería la

posición de una masa a la izquierda del primer resorte, en éste caso sería la pared, implica que:

$$
\mathrm{X}_{0}=\mathrm{X}_{00}=0
$$

$\mathrm{X}_{4}$ sería la posición de una masa a la derecha del último resorte, en éste caso sería la pared, implica que:

$$
\mathrm{X}_{4}=\mathrm{X}_{04}=\mathrm{L}
$$

Por comodidad se realiza un cambio de variable en el lagrangiano, a una variable que represente elongación del resorte, sea:

$$
Z_{j}=X_{j}-X_{0 j}
$$

Y sean $\boldsymbol{V}_{j}$ las variables correspondientes a $\mathrm{q}_{\mathrm{j}}$ en la transformación de coordenadas:

$\mathrm{L}\left(\mathrm{Z}_{\mathrm{j}}, v_{j}\right)=$

$\frac{1}{2} \sum_{j=1}^{3} M_{j} v_{j}^{2}-\frac{1}{2} \sum_{j=0}^{3} K_{j}\left(z_{j+1}-z_{j}\right)^{2}$ 
Usando la ecuación de Euler se obtienen las ecuaciones para $Z_{j}$ sobre la trayectoria:

$$
\left(\frac{\partial L}{\partial v_{j}}\right)^{\bullet}-\left(\frac{\partial L}{\partial z_{j}}\right)=0 \quad j=1,2,3
$$

Además considerando que todas las esferas tienen la misma masa " $\mathrm{M}$ " y todos los resortes tienen la misma constante de elasticidad " $\mathrm{K}$ " y siendo:

$$
w^{2}=\frac{K}{M}
$$

Entonces:

$$
\begin{array}{r}
\ddot{z}_{j}=w^{2}\left(z_{j-1}-2 z_{j}+z_{j+1}\right) \\
j=1,2,3
\end{array}
$$

Expandiendo las ecuaciones se tiene el sistema:

$$
\begin{aligned}
& w^{2}\left(0-2 Z_{1}+Z_{2}\right)=\ddot{Z}_{1} \\
& w^{2}\left(Z_{1}-2 Z_{2}+Z_{3}\right)=\ddot{Z}_{2} \\
& w^{2}\left(Z_{2}-2 Z_{3}+0\right)=\ddot{Z}_{1}
\end{aligned}
$$

De la transformación (5) y además de (3) y (4) se tiene que:

$$
Z_{0}=0 \quad \text { y } Z_{4}=0
$$

El sistema de ecuaciones (9), (10) y (11) puede llevarse a una ecuación matricial:

$$
\begin{gathered}
w^{2}\left(\begin{array}{ccc}
-2 & 1 & 0 \\
1 & -2 & 1 \\
0 & 1 & -2
\end{array}\right)\left(\begin{array}{l}
Z_{1} \\
Z_{2} \\
Z_{3}
\end{array}\right)=\left(\begin{array}{l}
Z_{1} \\
Z_{2} \\
Z_{3}
\end{array}\right)^{\bullet \bullet} \equiv \\
w^{2} A \vec{Z}=\vec{Z}^{\bullet \bullet}
\end{gathered}
$$

Donde si la matriz "A" fuese diagonal, el sistema de ecuaciones estaría resuelto. Por otro lado para que el modelo corresponda con el comportamiento experimental armónico, los valores de esa matriz diagonal deberían ser negativos.

Hallando los vectores y valores propios de A:

$$
A \vec{u}_{k}=\lambda_{k} \vec{u}_{k}
$$

Donde $\mathrm{k}$ es el número de valores propios del sistema, no debe confundirse con el número de esferas:

$$
U=\left(\begin{array}{lll}
\vec{u}_{1} & \vec{u}_{2} & \vec{u}_{3}
\end{array}\right)=\left(\begin{array}{ccc}
0.5000 & -0.7071 & -0.5000 \\
-0.7071 & 0.0000 & -0.7071 \\
0.5000 & 0.7071 & -0.5000
\end{array}\right)
$$

$$
\left(\lambda_{k}\right)=\left(\begin{array}{ccc}
\lambda_{1} & 0 & 0 \\
0 & \lambda_{2} & 0 \\
0 & 0 & \lambda_{3}
\end{array}\right)=\left(\begin{array}{ccc}
-3.4142 & 0 & 0 \\
0 & -2.0000 & 0 \\
0 & 0 & -0.5858
\end{array}\right)
$$

$$
A U=\left(\lambda_{k}\right) U
$$

Sea:

$$
\vec{S}=U^{-1} \vec{Z}
$$

Por tanto reemplazando (17) y (18) en la ecuación (13):

$$
w^{2} A U \vec{S}=(U \vec{S})^{\bullet \bullet}
$$

La matriz U no depende del tiempo:

$$
w^{2} U^{-1} A U \vec{S}=(\vec{S})^{\bullet \bullet}
$$

De (17) y (20) se tiene que: 


$$
w^{2}\left(\lambda_{k}\right) \vec{S}=(\vec{S})^{\bullet \bullet}
$$

Sea $w_{k}^{2}=w^{2} \lambda_{k}$ como $\lambda_{\mathrm{k}}$ es negativo, las soluciones para $\mathrm{S}$ son armónicas:

$$
S_{k}(t)=a_{k} \operatorname{Sen}\left(w_{k} t+b_{k}\right)
$$

$\mathrm{S}_{\mathrm{k}}$ son las coordenadas normales (o principales propias o propias) del sistema, no son las coordenadas coordenadas de las esferas. "a" y "b" son constantes y dependen de las condiciones iniciales.

Debido a (18) y (22) se puede escribir las funciones que representan a las elongaciones de los resortes como:

$$
\begin{aligned}
& Z_{1}(t)=U_{1 k} S_{k}(t)=\sum_{k=1}^{3} U_{1 k} a_{k} \operatorname{Sen}\left(w_{k} t+b_{k}\right) \\
& Z_{2}(t)=U_{2 k} S_{k}(t)=\sum_{k=1}^{3} U_{2 k} a_{k} \operatorname{Sen}\left(w_{k} t+b_{k}\right) \\
& Z_{3}(t)=U_{3 k} S_{k}(t)=\sum_{k=1}^{3} U_{3 k} a_{k} \operatorname{Sen}\left(w_{k} t+b_{k}\right)
\end{aligned}
$$

Las coordenadas físicas de las esferas serán:

$$
\begin{aligned}
& X_{1}(t)=Z_{1}(t)+X_{01}=X_{01}+\sum_{k=1}^{3} U_{1 k} a_{k} \operatorname{Sen}\left(w_{k} t+b_{k}\right) \\
& X_{2}(t)=Z_{2}(t)+X_{02}=X_{02}+\sum_{k=1}^{3} U_{2 k} a_{k} \operatorname{Sen}\left(w_{k} t+b_{k}\right) \\
& X_{3}(t)=Z_{3}(t)+X_{03}=X_{03}+\sum_{k=1}^{3} U_{3 k} a_{k} \operatorname{Sen}\left(w_{k} t+b_{k}\right)
\end{aligned}
$$

Como se observa de las ecuaciones, la oscilación de cada una de las esferas depende de las oscilaciones de sus vecinas, además sus condiciones iniciales.

Cuando se implementa el sistema experimentalmente y quiere darle una condición inicial. Debe separarse o unirse las esferas y luego ir soltando a cada una.

Las constantes $b_{k}$ son diferencias de fase que corresponden al instante inicial con la cual se van soltando las esferas. En el presente trabajo se considerará que en el instante $t=0 \mathrm{~s}$ se sueltan simultáneamente a las esferas, además para evitar que en el instante inicial no exista elongación, entonces $b_{k}=\pi / 2$.

Bajo dichas premisas y considerando:

$$
\vec{Z}(0) \cdot \vec{u}_{k}=\sum_{r=1}^{3} a_{r} \vec{u}_{r} \cdot \vec{u}_{k}=\sum_{r=1}^{3} a_{r} \delta_{k r}=a_{k}
$$

Por tanto, ya se tiene las ecuaciones de movimiento del sistema.

También es interesante que en la simulación se observen los estados propios del sistema descritos por:

$$
P_{k}(t)=U S_{k}(t)
$$

Los estados propios son modos de oscilación primigenios del sistema, a partir de los cuales se pueden crear todos los posibles modos en que oscilará el sistema mediante una combinación lineal de estos estados propios.

Los tres estados propios dan las ecuaciones para cada esfera y son:

Estado propio $\mathrm{P}_{1}$ :

$$
\begin{aligned}
& X_{1}(t)=X_{01}+\left(\vec{u}_{1}\right)_{1} \operatorname{Sen}\left(w_{1} t+b_{k}\right) \\
& X_{2}(t)=X_{02}+\left(\vec{u}_{1}\right)_{2} \operatorname{Sen}\left(w_{1} t+b_{k}\right) \\
& X_{3}(t)=X_{03}+\left(\vec{u}_{1}\right)_{3} \operatorname{Sen}\left(w_{1} t+b_{k}\right)
\end{aligned}
$$

Estado Propio $\mathrm{P}_{2}$ :

$$
X_{1}(t)=X_{01}+\left(\vec{u}_{2}\right)_{1} \operatorname{Sen}\left(w_{2} t+b_{k}\right)
$$




$$
X_{2}(t)=X_{02}+\left(\vec{u}_{2}\right)_{2} \operatorname{Sen}\left(w_{2} t+b_{k}\right)
$$

Estado propio P3:

$$
\begin{aligned}
& X_{1}(t)=X_{01}+\left(\vec{u}_{3}\right)_{1} \operatorname{Sen}\left(w_{3} t+b_{k}\right) \\
& X_{2}(t)=X_{02}+\left(\vec{u}_{3}\right)_{2} \operatorname{Sen}\left(w_{3} t+b_{k}\right) \\
& X_{3}(t)=X_{03}+\left(\vec{u}_{3}\right)_{3} \operatorname{Sen}\left(w_{3} t+b_{k}\right)
\end{aligned}
$$

La notación $\left(\vec{u}_{i}\right)_{j}$ significa la j-ésima componente del vector $\vec{u}_{i}$.

$$
X_{3}(t)=X_{03}+\left(\vec{u}_{2}\right)_{3} \operatorname{Sen}\left(w_{2} t+b_{k}\right)
$$

\section{EXPERIMENTO}

Los experimentos virtuales se hicieron en el programa Adobe Flash Professional 8.

Experimento virtual: Determinación del valor de la gravedad terrestre.

Se abrió un documento flash en blanco y se dimensionó el lienzo de trabajo a un tamaño de 550x550 pixel, como se muestra en la Figura 3.

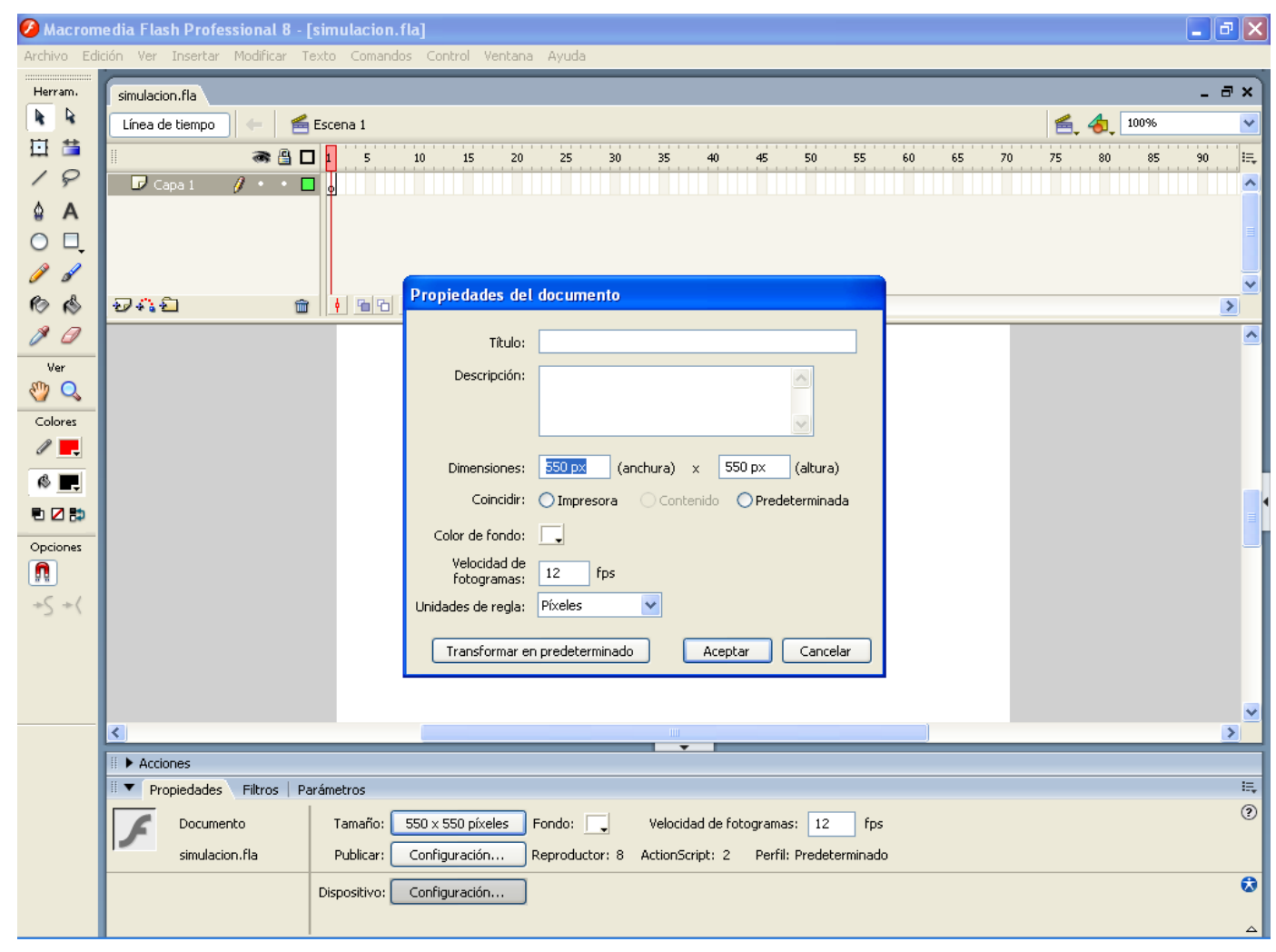

Figura 3. Dimensionamiento del lienzo de trabajo.

Se importó al escenario la imagen, de una de una regla graduada en $\mathrm{cm}$.

La regla se colocó verticalmente, se editó en propiedades la posición y ancho a: X: 0.0 Y: 0.0 con an: 96 alt: 550. Se dibujó una línea de color negro y ancho 1pixel, desde el extremo superior derecho de la regla hacia el extremo superior izquierdo del lienzo de trabajo. Como se muestra en la Figura 4. 


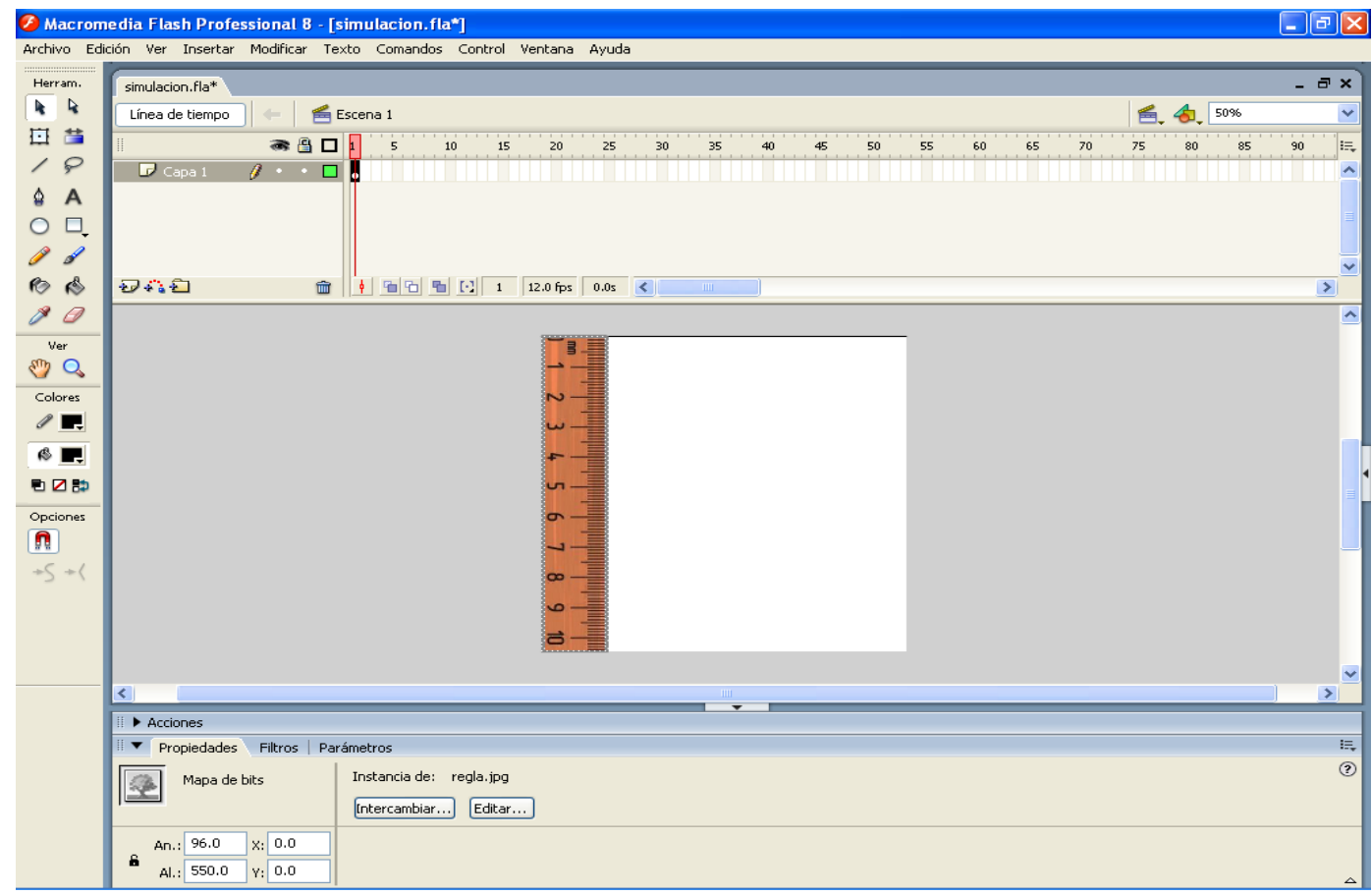

Figura 4: Trazo de una línea negra de ancho 1 pixel sobre el lienzo de trabajo.

Se importó la imagen de un resorte al escenario, se ubicó en la parte superior del lienzo, de tal manera que uno de sus extremos esté muy cercano a la línea negra. Además haciendo clic derecho sobre el resorte, se seleccionó la opción convertir en símbolo. Éste procedimiento de muestra en la Figura 5.

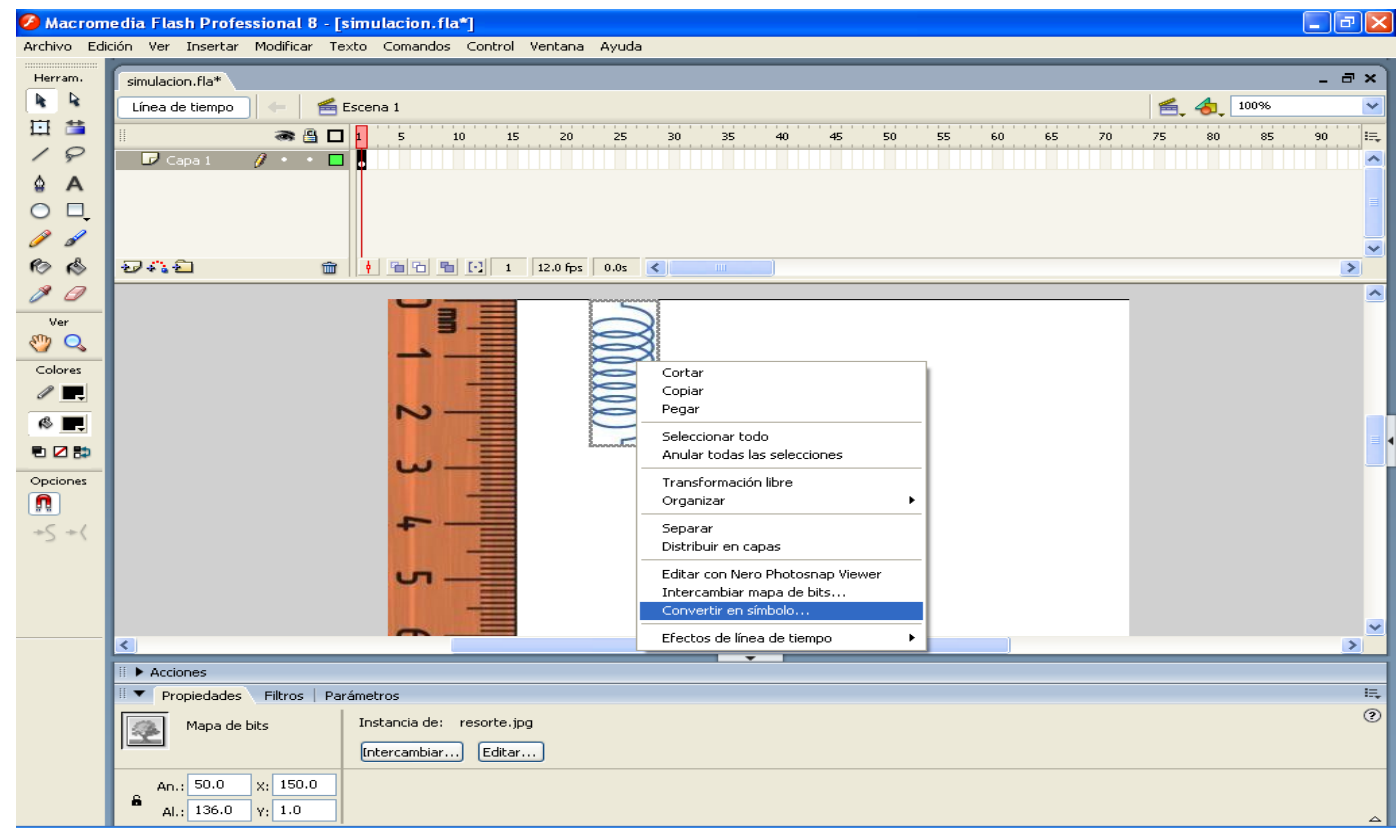

Figura 5. Conversión de la imagen del resorte en símbolo. 


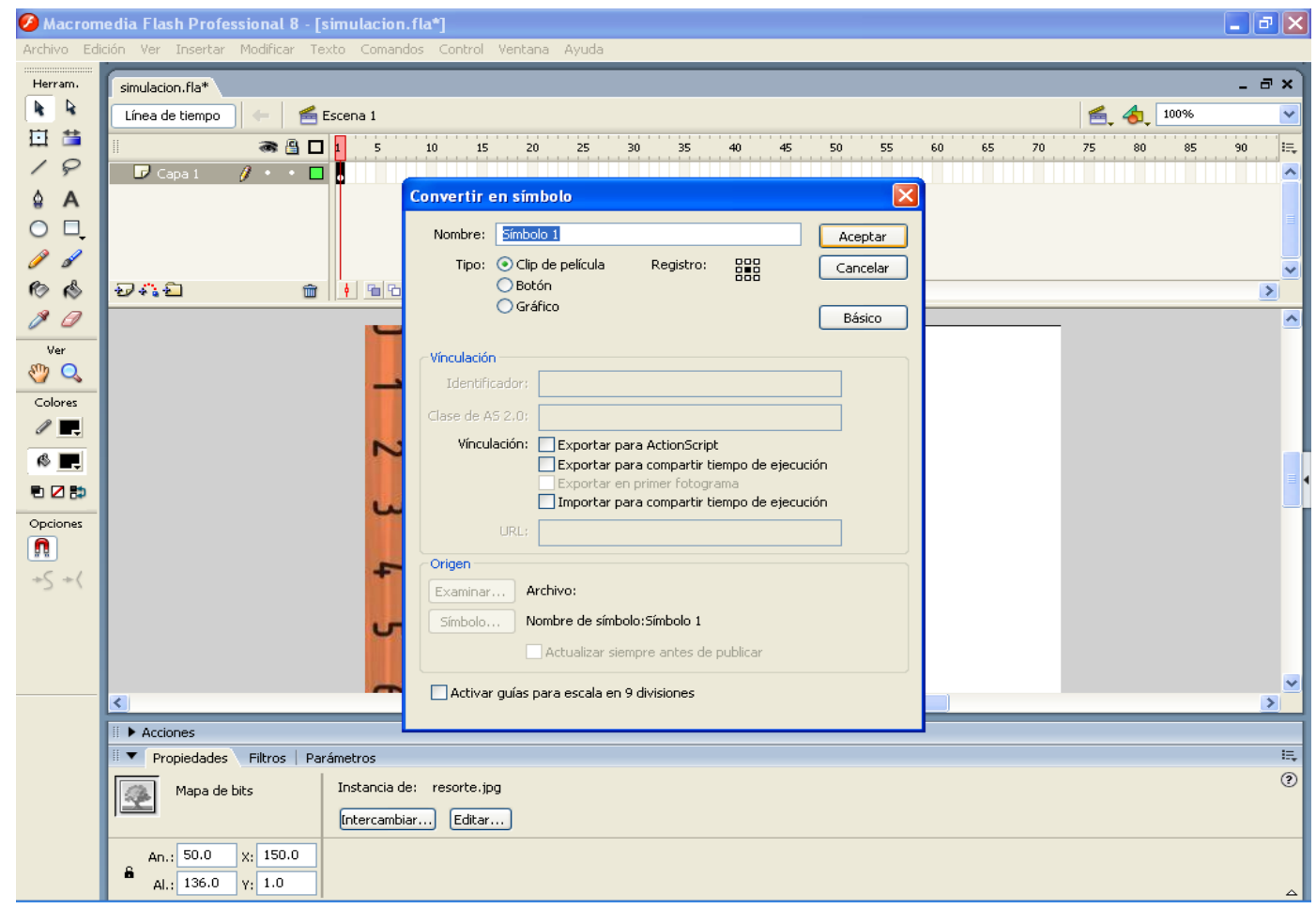

Figura 6. Conversión en símbolo de tipo clip de película.

Posteriormente se colocó en propiedades, en nombre de la instancia, el nombre de variable con la cual será reconocido el resorte durante todo el código en ActionScript. En éste caso el nombre de la variable fue "r", ver Figura 7.

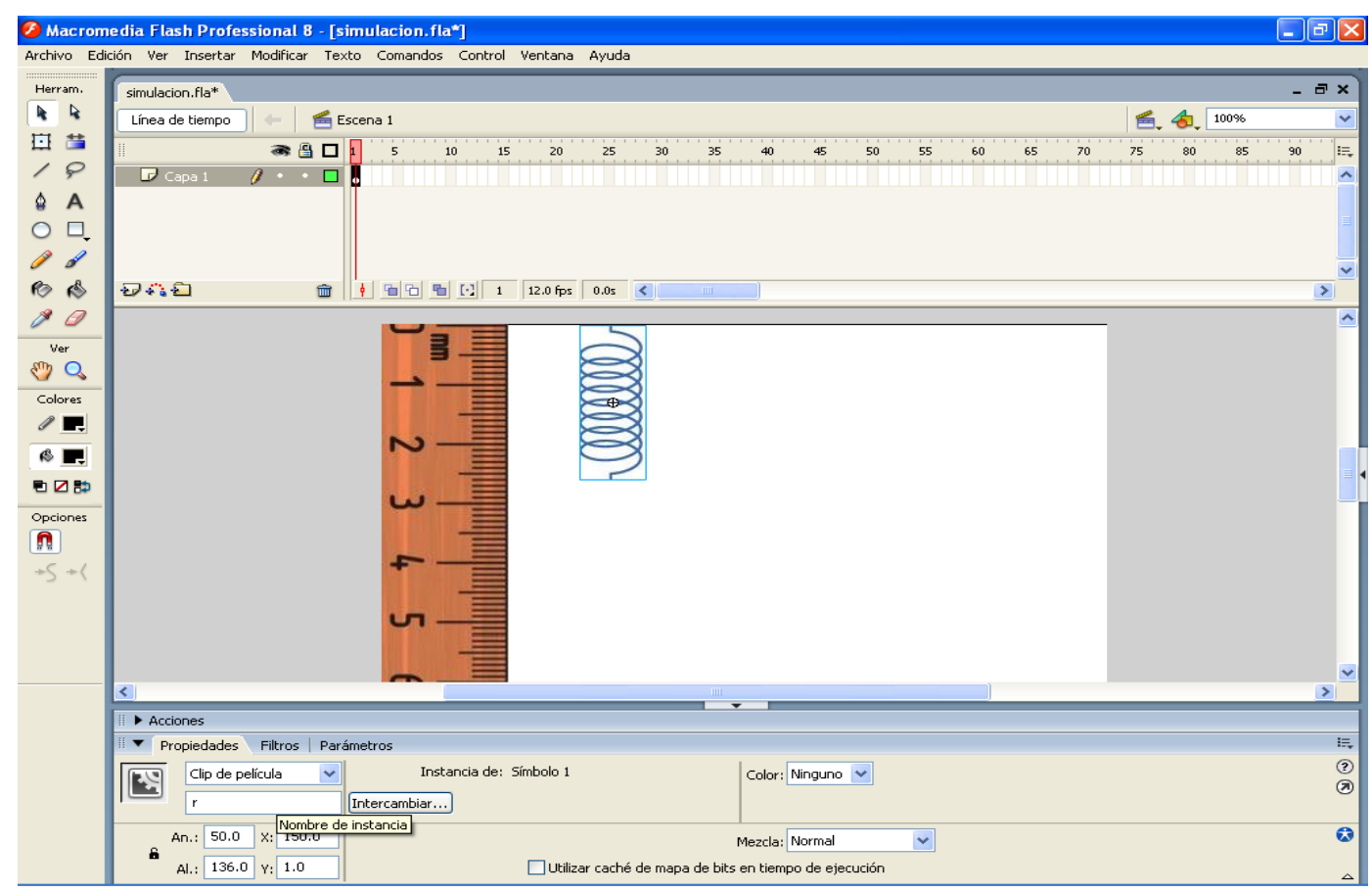

Figura 7: Ingreso del nombre de instancia " $r$ " en la opción propiedades. 
Se dibujó una esfera que está colgando del resorte la cual posee unida a ella una varilla muy delgada sin peso que servirá solo como
Indicador. Se agruparon y al conjunto se le convirtió en clip de película. Se le colocó como nombre de instancia "c". Como se muestra en la Figura 8.

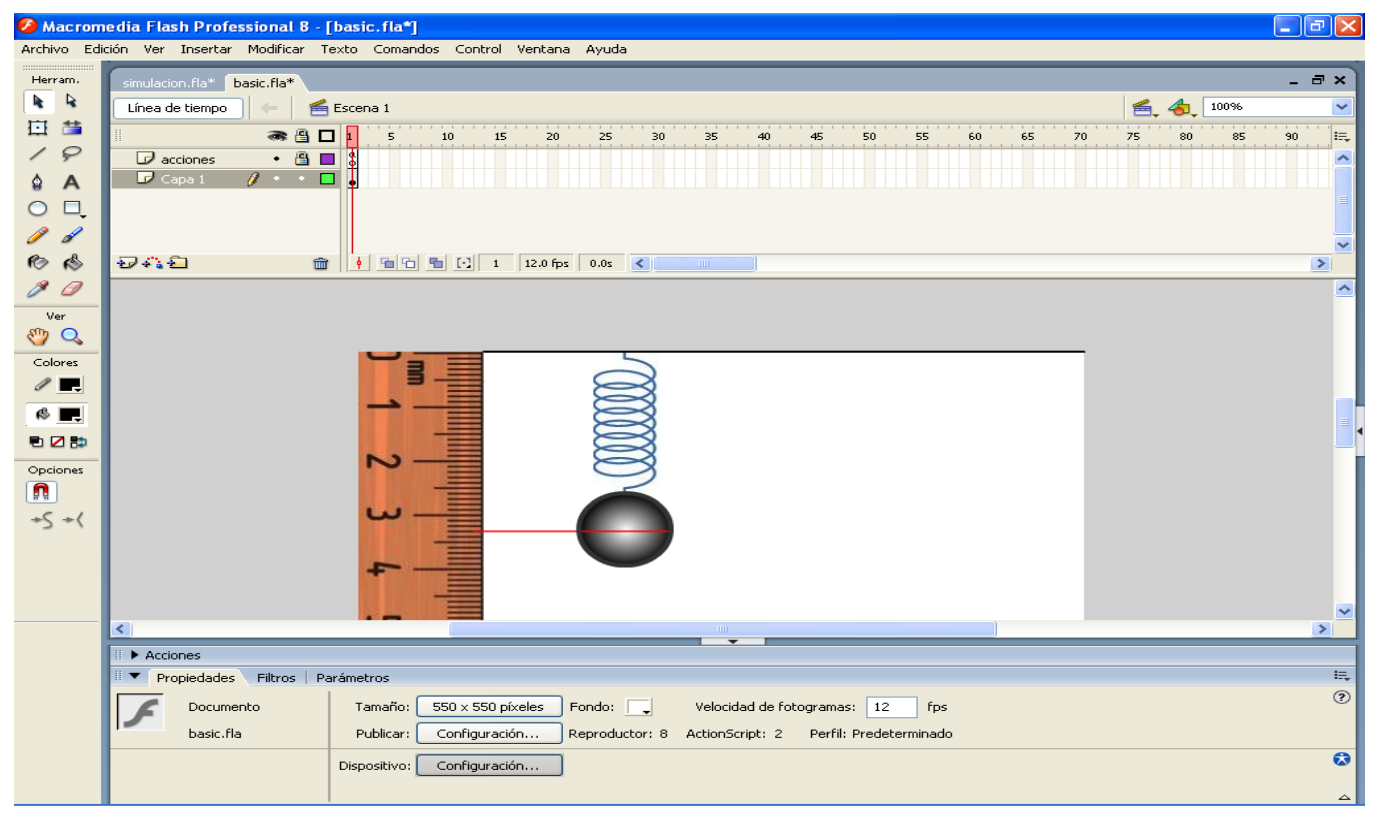

Figura 8. Dibujo de una esfera colgado del resorte.

Luego se agregaron textos estáticos indicando el título del experimento, las unidades y nombres de las variables a introducir.

Además se colocaron dos recuadros para la introducción de texto. Estos recuadros sirven para introducir los valores de K y M. A éstos recuadros de introducción de texto se les pusieron como nombre de instancia " $\mathrm{k}$ " $\mathrm{y}$ " $\mathrm{m}$ ". Ver Figura 9.

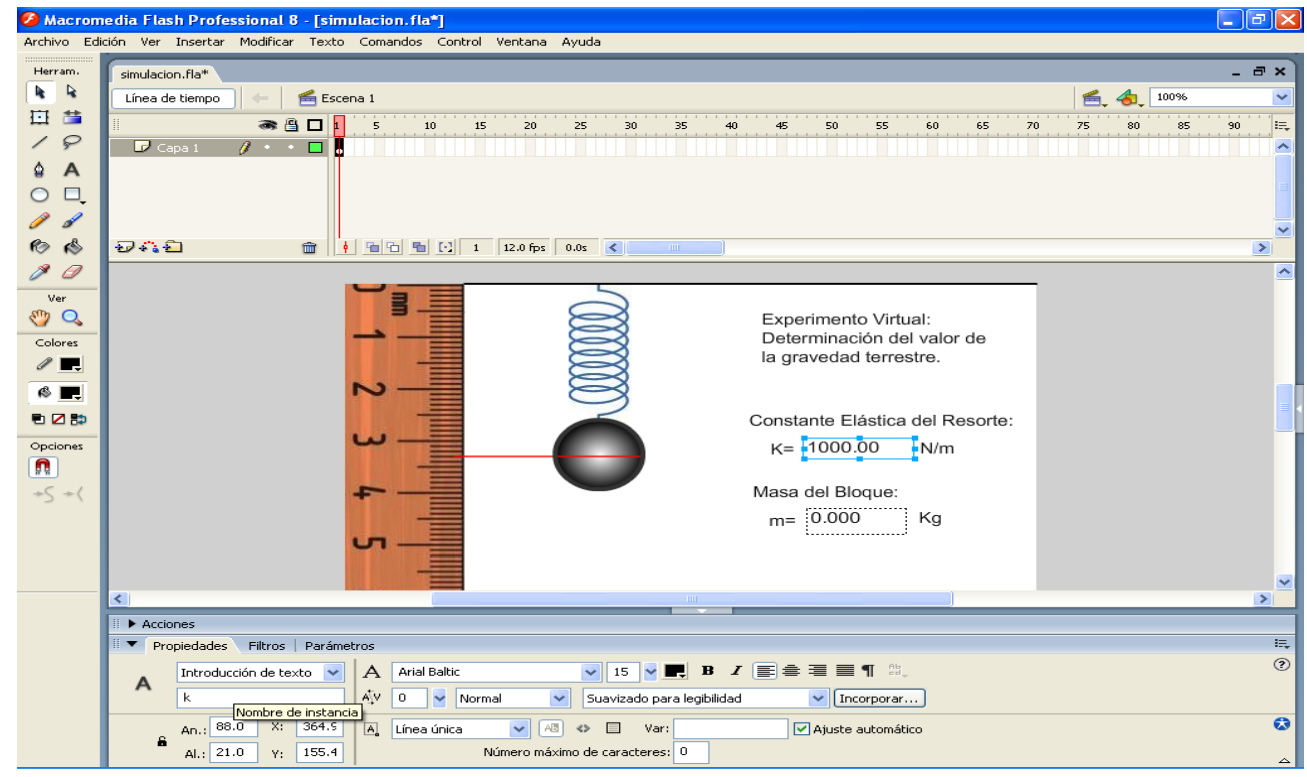

Figura 9. Introducción de los valores de $K$ y M. 
Se importaron dos imágenes de botones para que controlen el inicio y final de la ejecución del programa.
Las imágenes se convirtieron en clip de película y se le pusieron como nombre de instancia "b1" y "b2". Ver Figura 10.

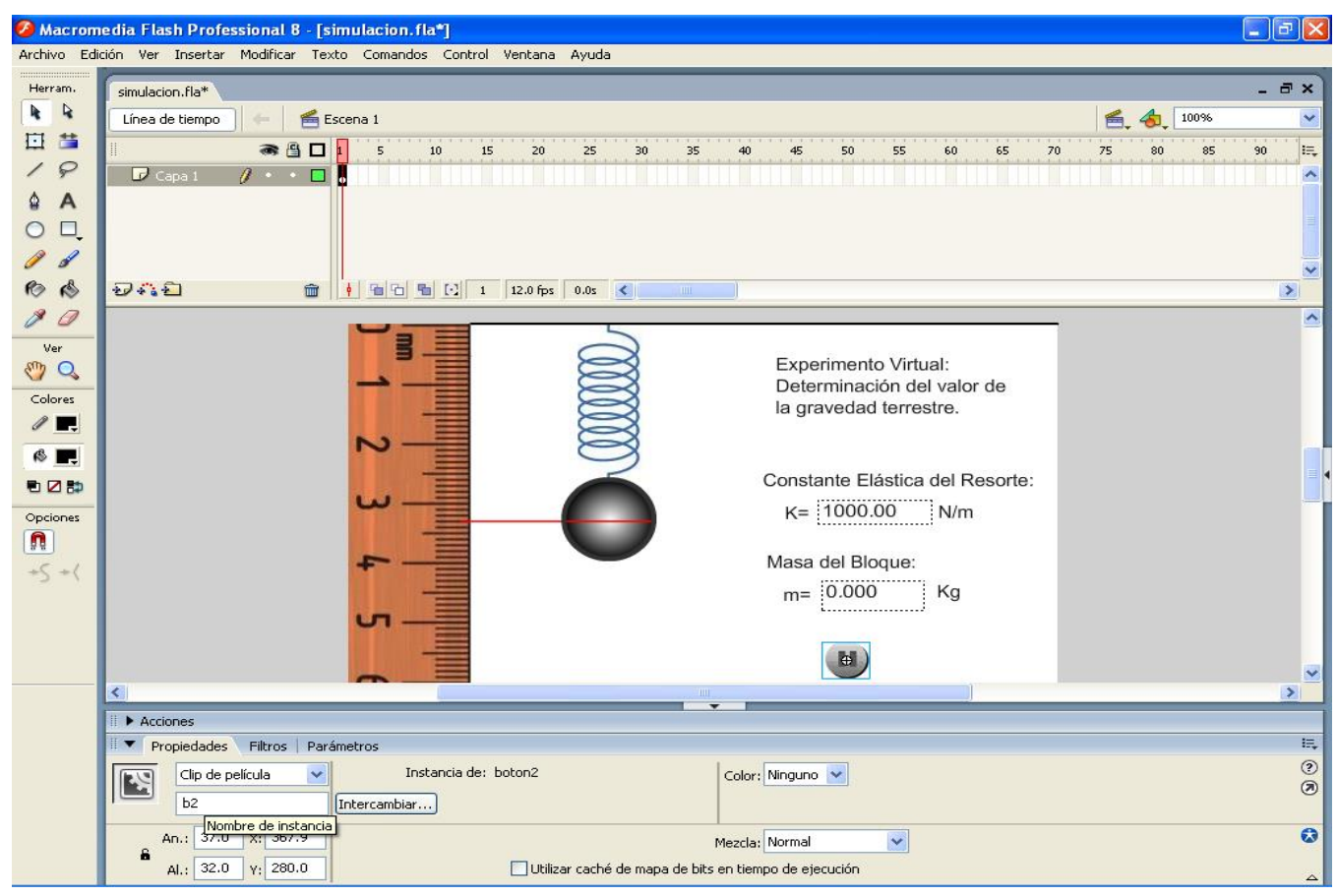

Figura 10. Conversión en clip de película de las imágenes de botones importadas.

Luego se agregó una capa nueva, se la llamó "acciones", en ella se programará las acciones que tendrán cada uno de los clips de película.
En esa capa se colocó las Acciones del ActionScript como se observa en la Figura 11.

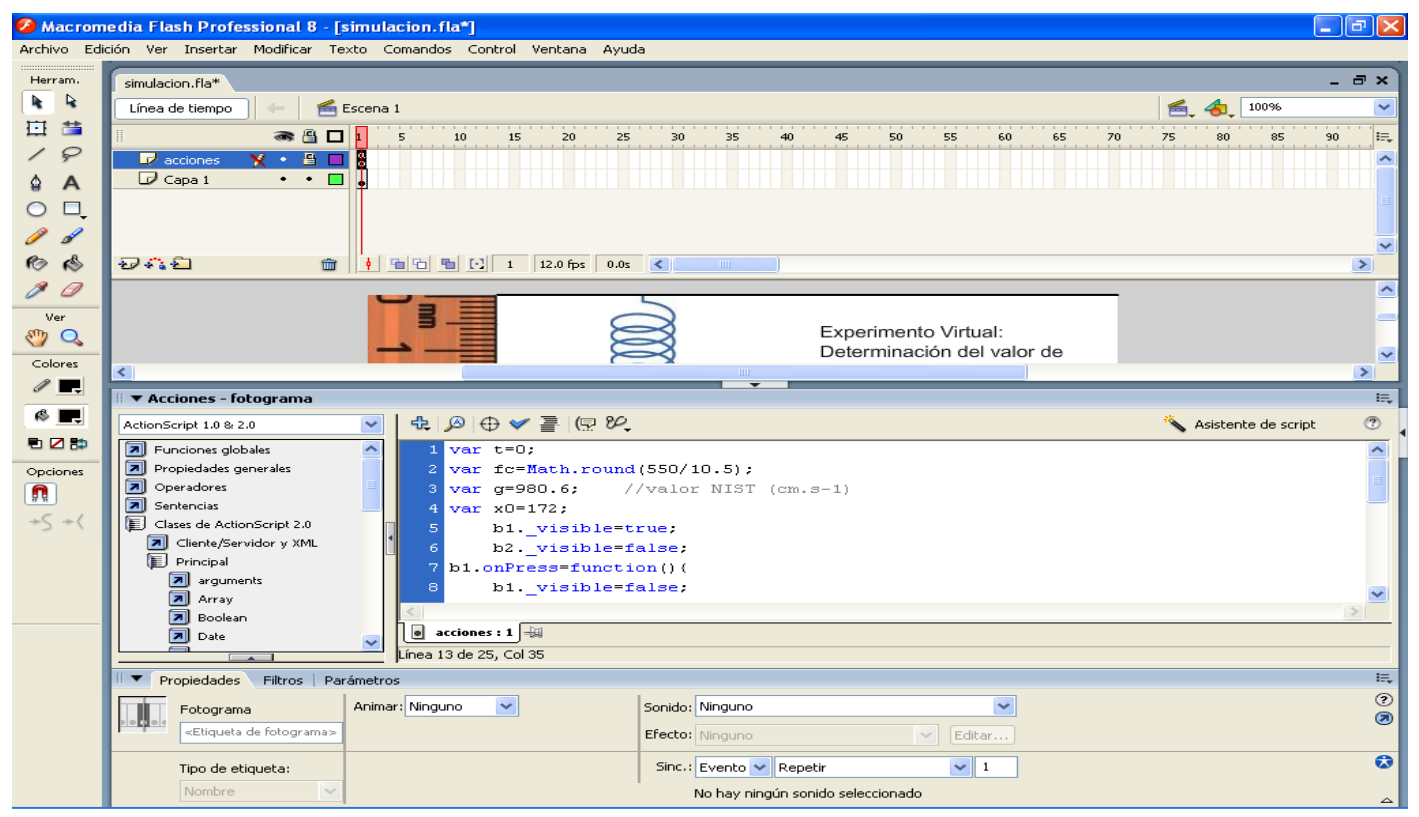

Figura 11. Programación de las acciones para cada uno de los clips de película. 
Usando la fórmula (1), se desarrolló el código del programa en ActionScript:

var $\mathrm{t}=0$;

var $\mathrm{fc}=$ Math.round $(550 / 10.5)$;

var $\mathrm{g}=980.6 ; \quad / /$ valor NIST (cm.s-1)

var $\mathrm{x} 0=172$;

b1._visible=true;

b2._visible $=$ false;

b1.onPress=function ()\{

b1._visible $=$ false;

b2._visible=true;

var $\mathrm{K}=\mathrm{k}$.text;

var $\mathrm{M}=\mathrm{m}$.text;

OnEnterFrame $=$ function ()\{

c. $\mathrm{y}=\mathrm{x} 0+\mathrm{Math}$.round $(\mathrm{fc} *((\mathrm{~g} * \mathrm{M}) / \mathrm{K}))$;

r._height=Math.Round $((\mathrm{c}$._y-c._height/2)));

b2._Visible=false; b1. Visible=true;

OnEnterFrame=function () \{stop ();

\}<smiles>I[IH]</smiles>

Se compilo el programa y se obtuvo el archivo en extensión SWF el cual puede ejecutarse fácilmente sin necesidad de instalar el Adobe Flash 8,o se puede incluir en una página web para su ejecución por cualquier persona que accede a dicha página web, independientemente del Sistema operativo que use .Este hecho ofrece una gran posibilidad de que los alumnos en Universidades o en colegios que estén conectados a internet como resultado de la política de gobierno de una computadora por niño, usen este tipo de herramientas en su proceso formativo.

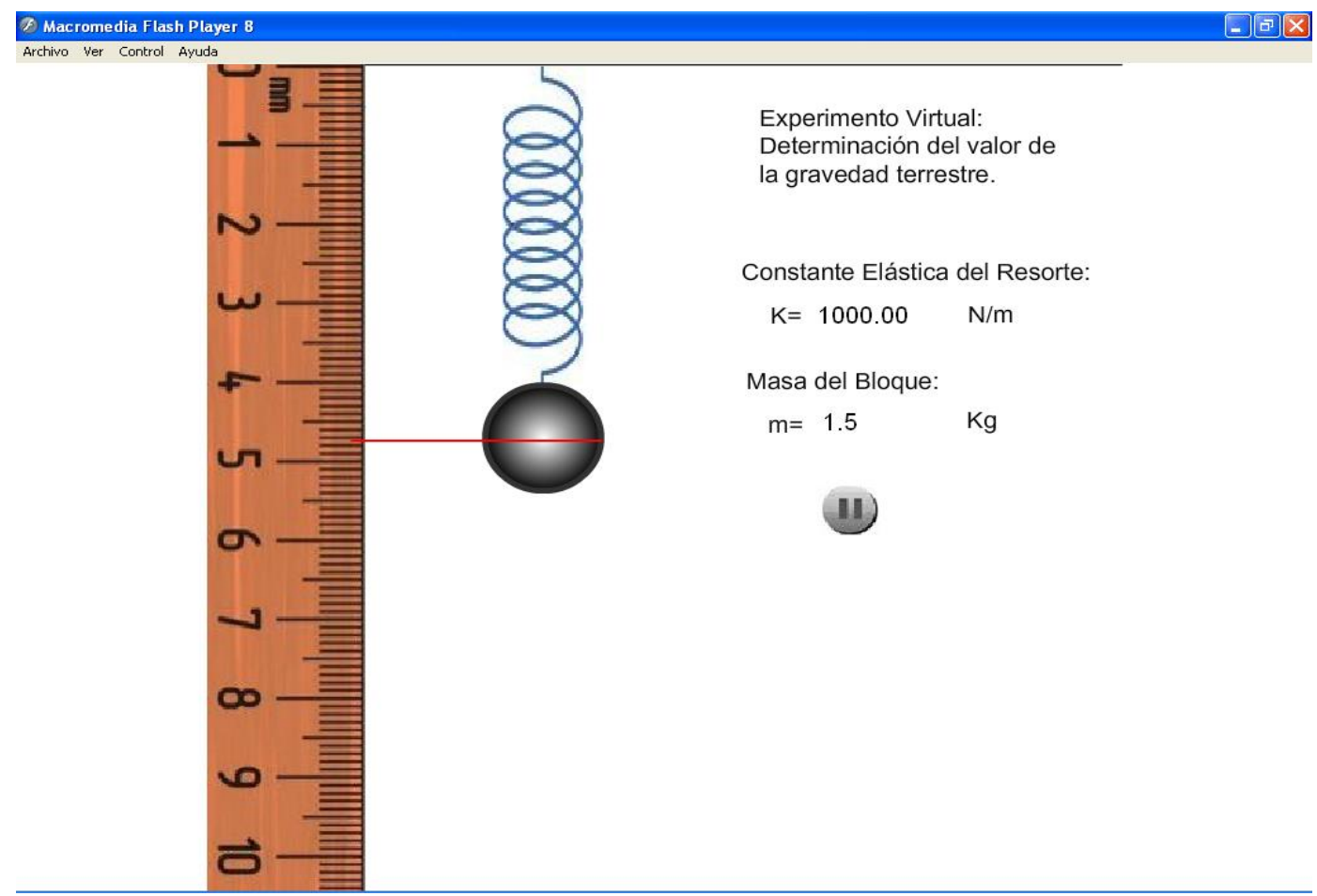

Figura 12. Primer Experimento Virtual en ejecución.

Los usuarios del experimento virtual pueden cambiar los valores de $\mathrm{K}$ y $\mathrm{m}$ para hallar $\mathrm{g}$.
Por ejemplo haciendo diez mediciones, con $\mathrm{K}=1000.00 \mathrm{~N} / \mathrm{m}$ y variando " $\mathrm{m}$ " desde $0.5 \mathrm{Kg}$ hasta $5.0 \mathrm{Kg}$ en incrementos de $0.5 \mathrm{Kg}$, se obtuvo la Tabla1. 
Tabla1. Valores hallados con el experimento virtual.

\begin{tabular}{|c|c|c|}
\hline Masa $(\mathrm{kg})$ & Elongación $(\mathrm{m})$ & $\mathrm{g}\left(\mathrm{m} . \mathrm{s}^{-2}\right)$ \\
\hline 0.5 & 0.005 & 10.0 \\
\hline 1.0 & 0.010 & 10.0 \\
\hline 1.5 & 0.015 & 10.0 \\
\hline 2.0 & 0.019 & 9.5 \\
\hline 2.5 & 0.024 & 9.6 \\
\hline 3.0 & 0.029 & 9.7 \\
\hline 3.5 & 0.034 & 9.7 \\
\hline 4.0 & 0.039 & 9.8 \\
\hline 4.5 & 0.043 & 9.6 \\
\hline 5.0 & 0.048 & 9.6 \\
\hline & & 9.7 \\
\hline & $\bar{g}$ & \\
\hline
\end{tabular}

El valor de gravedad hallado con el experimento virtual es coherente con el valor estándar de $9.8 \mathrm{~ms}^{2}$.

Experimento virtual: Sistema 3 masas 4 resortes.
Siguiendo los mismos pasos descritos en el punto 3.1 y usando las fórmulas desde la (26) hasta la (39) se desarrolló el experimento que se muestra en la Figura 13.

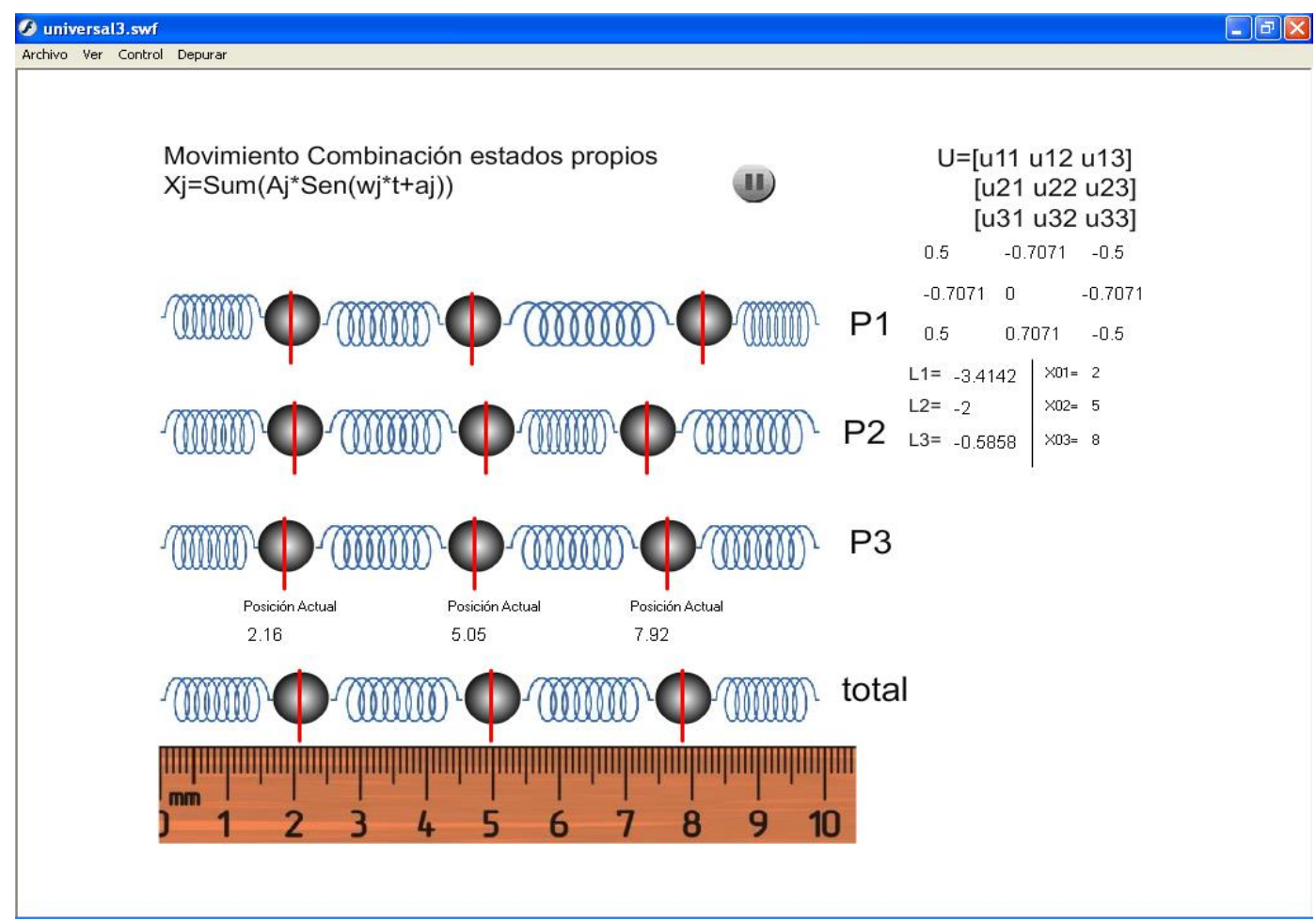

Figura 13. Segundo Experimento Virtual en ejecución. 
Se usó una regla horizontal para tener una referencia en el movimiento, se consideró que las masas de las esferas son de $1 \mathrm{Kg}$ y la constante elástica de los resortes $\mathrm{K}=1 \mathrm{~N} / \mathrm{m}$.

Se pudo observar como los estados propios determinan el movimiento complejo final del sistema.

Se colocaron indicadores para saber la posición exacta de las posiciones de las esferas en el movimiento total.

El usuario puede interactuar con el experimento virtual cambiando los valores de la matriz de valores propios, así como los vectores propios y las posiciones iniciales.

\section{CONCLUCIONES}

Se hizo un experimento virtual que permite que el estudiante pueda calcular el valor de la gravedad terrestre, así como tenga una noción visual de lo que es la constante elástica de un resorte y cuál es su efecto sobre el mismo, cuando se le aplica una determinada fuerza.

El experimento virtual es interactivo, permite que el estudiante cambie los valores de la constante elástica del resorte y de la masa que sostiene.

Usando el experimento virtual y considerando $\mathrm{K}=1000 \mathrm{Nm}^{-1}$ y masas desde $0.5 \mathrm{Kg}$ hasta $5.0 \mathrm{Kg}$ en incrementos de $0.5 \mathrm{Kg}$, se encontró que el valor de la aceleración de la gravedad fue de $(9.7 \pm 0.2) \mathrm{ms}^{-2}$, lo cual es coherente con el valor estándar de $9.8 \mathrm{~ms}^{-2}$.

Se hizo un experimento virtual que permite simular un sistema de tres masas unidas por cuatro resortes. El experimento virtual no solo permite visualizar el movimiento del sistema, sino que también permite ver los movimientos propios del sistema.

El experimento virtual es interactivo, permite que el estudiante cambie los valores de la matriz de valores propios, los vectores propios y las posiciones iniciales.
Los experimentos virtuales pueden usarse como herramienta para permitir que los estudiantes tomen un rol activo en una clase interactuando con el modelo y luego formulando preguntas al profesor acerca de las dificultades sobre el entendimiento del fenómeno.

Económicamente es una herramienta absolutamente accesible a gran cantidad de estudiantes, pues al ser Adobe Flash un aplicativo pensado para ejecutarse desde una página web, permite que muchos estudiantes usen los experimentos virtuales al mismo tiempo, sin importar su sistema operativo ni su ubicación geográfica.

\section{AGRADECIMIENTO}

El presente artículo originalmente fue un trabajo hecho durante mis estudios de Maestría en Física en la Facultad de Ciencias de la Universidad Nacional de Ingeniería, en el curso de Mecánica Clásica dictado por el Profesor Holguer Valqui Casas. Conversando con el Profesor Valqui acerca de la publicación del reporte a través de un artículo, se mostró entusiasmado, sin embargo, no deseaba tener una co-autoría pero si que se mencionase que las ecuaciones usadas para los programas fueron tomadas de: "los apuntes de clase de hv". Le agradezco mucho al Prof. Valqui por su atención y apoyo durante todo el curso y después del mismo.

Agradezco también a la Bach. Yovitza Lucia Romero Ramos por su apoyo en la edición de la última versión del escrito.

\section{BIBLIOGRAFÍA}

1. Hake, Richard.R., Am. J. Phys. 66 (1), January 1998

2. McDermott Lilian.C. , Redish Edgard F, Am. J. Phys. 67 (9), September 1999

3. Waltner Christine, Wiesner Hartmut and Rachel Alexander Phys Educ 42 (5) 2007. 
4. Meltzel David E., Manivannan Kandiah, Am. J. Phys. 70 (6), June 2002.

5. Borghi L, De Ambrosis A, Mascheretti $\mathbf{P}$ and Massara C I Phys Educ 22 (1) 1987.

6. Zacharias Zachariaa O. Anderson Roger Am. J. Phys. 71 (6), June 2003.

7. Lee Koo-Chul, Lee Julian, Computer Physics Communications 177 (2007) 195-198.

8. Mihas Pavlos. Phys Educ 38 (1) 2003

9. Humberston $\mathbf{J} \mathbf{W}$, McKenzie $\mathbf{J}$ and McTiernan P G Phys Educ., Vol 18, 1983

10. Rubio Garcia Ramon; Suarez Quiros, Javier; Gallego Santos Ramon; Martın Gonzalez Santiago; Moran Fernanz Samuel. Computers \& Education 50 (2008) 772-786 